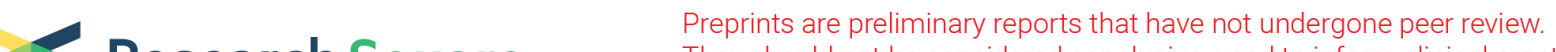 Research Square They should not be considered conclusive, used to inform clinical practice, or referenced by the media as validated information.
}

\section{Abdominopelvic leiomyoma with large ascites: a case report and literature review}

\section{Yiwei Wang}

Shanghai Jiaotong University: Shanghai Jiao Tong University

Qiong Fan

Shanghai Jiaotong University: Shanghai Jiao Tong University

\section{Zhaoxia Qian}

Shanghai Jiaotong University: Shanghai Jiao Tong University

Jinjin Wang

Shanghai Jiaotong University Pao Yue Kong Library: Shanghai Jiao Tong University

\section{Yuhong Li}

Shanghai Jiao Tong University School of Medicine

Yudong Wang ( $D$ owangyudong@126.com )

Shanghai Jiao Tong University https://orcid.org/0000-0003-4251-4109

\section{Case report}

Keywords: leiomyoma, ascites, greater omentum, pseudo-Meigs syndrome

Posted Date: September 24th, 2020

DOl: https://doi.org/10.21203/rs.3.rs-80944/v1

License: (c) (1) This work is licensed under a Creative Commons Attribution 4.0 International License. Read Full License 


\section{Abstract}

\section{Background}

Leiomyoma of the uterus is relatively common, but uterine leiomyoma of the greater omentum is rare.

Case presentation

Here, we report a case of a 22-year-old woman who presented with a 3-month history of progressive abdominal distension and a hypervascular abdominopelvic mass. Due to a high serum concentration of CA125, the preoperative diagnosis was unclear. During surgery, $5 \mathrm{~L}$ of ascites was removed. An $18.8-\mathrm{cm}$ solid mass, which was pedunculated from the uterine fundus and exhibited complex adhesion to the greater omentum, was removed. The CA125 level was reduced postoperatively, and a pathologic study confirmed that the mass was a leiomyoma that originated in the uterus.

\section{Conclusion}

Uterine leiomyoma can share vessels with the greater omentum. This case highlights the difficulty of diagnosing pseudo-Meigs syndrome and the importance of imaging and laboratory examinations.

\section{Background}

Uterine leiomyomas are common benign smooth muscle tumors that affect up to $80 \%$ of women [1-2]. Common uterine leiomyomas principally result from the estrogen- and progesterone-dependent transformation of uterine smooth muscle [3]. Affected women may complain of bleeding, pain, pressure or infertility, but few eventually develop uterine sarcoma [4-5].

Meigs syndrome is a clinical triad whose pathology is limited to ovarian fibroma, fibrothecoma, Brenner tumor and granulosa cell tumor [6-7] and is a syndrome characterized by ascites and hydrothorax. Pseudo-Meigs syndrome is defined as a disorder similar to Meigs syndrome, in which neoplasms of a different histological types, including uterine leiomyomas, are present [8-10]. Uterine leiomyomas that present as pseudo-Meigs syndrome have already been described in the literature [11-14]. However, few cases of pseudo-Meigs syndrome in which a leiomyoma is implanted on the omentum have been reported [15]. In this study, we report a massive abdominopelvic leiomyoma with large ascites in a 22year-old woman.

\section{Case Presentation}

A 22-year-old woman presented to the outpatient unit with abdominal pain. She was not married and had never been pregnant. She reported no recent trauma, fall, or sexual intercourse. She reported a normal menstrual cycle with a menstrual period eighteen days prior to her visit. The patient also reported a 3month history of increased abdominal circumference, which she considered a "pot belly". The abdomen was gravid, soft, and firm. A rectovaginal examination revealed a movable mass at the front of the uterus. 
The patient's laboratory test results are shown in Table 1. Unexpectedly, the CA125 level was elevated to $569.5 \mathrm{U} / \mathrm{ml}$. Computed tomography (CT) of the pelvis was performed after administration of an intravenous contrast agent (Fig. 1), and magnetic resonance imaging (MRI) (data not shown) revealed a large mass with a maximum cross-section of $18.8 \times 13.5 \mathrm{~cm}$. Massive abdominal edema was also recorded. No evidence of swollen lymph nodes was found. Computed tomography angiography (CTA) revealed an abundant blood supply to the mass, which was provided by the uterine artery, superior mesenteric artery, and predominantly, by the gastroduodenal artery (Fig. 2). A review of systems was notable for findings of abdominal pain and a lack of appetite, but the woman denied clinical indications of constipation, diarrhea, vaginal bleeding, nausea, emesis, or urinary urgency or frequency.

The patient underwent abdominal aortic balloon catheter placement in case of hemorrhage and later underwent exploratory laparotomy under combined spinal-epidural anesthesia. Upon entering the abdomen, up to $5,000 \mathrm{ml}$ of yellow ascites was removed by suction (Fig. 3A). Ascitic fluid cytology revealed aggregated cells with inconspicuous atypia. A large solid cystic neoplasm that measured almost $20 \mathrm{~cm}$ was also found (Fig. 3B). In addition, careful inspection after ligation of the omental vessels showed that the omental fat had vanished and that vessels from the greater omentum, which were shared with the mass, were engorged. Adhesions between the mass and the ileocecum, appendix and descending colon were observed. The mass was found to be attached to the uterine fundus by a 3-cmwide pedicle (Fig. 3C). Resection of the mass and appendix was then performed (Fig. 3D), and during surgery, the total blood loss was $1,200 \mathrm{ml}$. The patient was transferred to recovery in stable condition, and on postoperative day 8 , she was discharged from the hospital.

A pathologic study of the specimen confirmed a diagnosis of leiomyoma. Hydropic degeneration, hyaline degeneration and cystic degeneration were recorded. An immunohistochemical analysis showed that the leiomyoma cells were positive for progesterone receptor, estrogen receptor, h-caldesmon and smooth muscle actin (Fig. 4). The tissue was negative for CD10, CD117, HMB45, Dog-1 and Ki67. The mass was determined to be a uterine-derived leiomyoma that had implanted on the omentum majus.

\section{Discussion And Conclusions}

The unique features of the presented case can be summarized as follows: a massive leiomyoma with the "digested" greater omentum and an elevated CA125 level accompanied by pseudo-Meigs syndrome. Uterine leiomyoma is the most common benign gynecological neoplasm [16]. However, elevated CA125 levels, a massive leiomyoma and large ascites, which is collectively known as pseudo-Meigs syndrome, are rarely seen in this condition [11-14, 17-20]. In addition, no agreement on the pathogenesis of this rare condition has been established.

In this case, the increased abdominal circumference was considered to have resulted from pleural effusion and ascites. The pathogenesis of ascites in pseudo-Meigs syndrome is still not well defined [21]. According to several hypotheses, the release of inflammatory cytokines and growth factors may cause vascular permeability [22-24]. An imbalance among arterial flow, venous drainage and direct pressure of 
the mass itself on lymphatic vessels may have caused the accumulation of nearly $5 \mathrm{~L}$ of ascites fluid in this case [25].

Removal of the large leiomyoma could resolve the ascites, but it is difficult to perform a complete excision when a leiomyoma is broadly adhered to adjacent structures. Full preparation, including detailed information about the size of the mass and its properties, could help in the design of a better surgical strategy [26]. Specifically, various imaging techniques, including CT and MRI, were used in this case, and both modalities indicated an 18-cm mass with abdominal edema.

CTA confirmed an abundant blood supply from the mass. During the surgery, distended blood vessels from the omentum (top), uterus (bottom) and colon (lateral) were observed and carefully ligated. Notably, the uterovesical peritoneal reflection was lifted, which was evidently due to the massive leiomyoma. Thus, preparations should have been made to reduce the risk of injury and to prevent uncontrolled bleeding or incomplete resection. Therefore, we performed abdominal aortic balloon catheter placement before surgery [27].

The elevated CA125 level is another complicating factor in this case. It is well known that as a high molecular weight glycoprotein, CA125 is expressed in a substantial proportion of ovarian cancers [28]. It has been reported that the serum CA125 levels range from 20 to over $1,000 \mathrm{IU} / \mathrm{ml}$ in patients with leiomyomas [29-31]. Due to the significant overlap between malignancies and myomas, CA125 failed to provide a potential benefit for differential diagnosis [32]. The destruction of the peritoneum and severe adhesions of the omentum could explain why CA125 was overexpressed before surgery and reduced after surgery [33-34].

A preoperative diagnosis of a pelvic mass with pseudo-Meigs syndrome remains very challenging, especially in cases such as the one presented in this study. In these cases, an accurate diagnosis may only be made after surgery. For oncological safety, patients with myomas may undergo hysterectomy or ovariectomy, which impair reproductive function. On the contrary, an overly optimistic presumption of a benign leiomyoma may lead to the intra-abdominal spread of an unexpected sarcoma, which could result in poor survival. Therefore, it is always important to consider a broad differential diagnosis and employ the necessary scrutiny using imaging techniques. Uterine sarcomas are rare gynecological neoplasms, and some researchers believe that sarcomas originate from benign leiomyomas because they contain the same epithelial progenitor cells [35]. Currently, no diagnostic technique is available to accurately distinguish this neoplasm. A meta-analysis revealed that expression of CA125, LDH and GDF-15 proteins is altered in sarcomas and that their serum levels are statistically higher [36]. In our case, the CA125 level was elevated. However, we interpreted this finding and believed the higher level was due to the implantation of the leiomyoma on the omentum rather than as a cancer-related signal. Deficiency of LDH and GDF-15 levels was not assessed in our case. In addition, MRI may help in the preoperative distinction of sarcomas from benign leiomyomas [37]. In our case, MRI revealed no swollen lymph nodes. However, the abundant blood supply to the mass observed on CTA imaging still roused our suspicion of a malignancy. Adenomyosis is another differential diagnosis of leiomyoma. A recent study has shown that 
MRI and elevated CA125 levels are fairly accurate for the diagnosis of adenomyosis, with a high sensitivity and specificity [29]. A mean serum CA125 level of $19 \mathrm{U} / \mathrm{ml}$ has been established as the cutoff value. We speculated that this cutoff did not apply to our case because of the size of the mass and the tight adhesion. Preoperative MRI revealed a suspected abdominopelvic mass with large ascites, which did not support the diagnosis of adenomyosis.

In conclusion, we have presented an unusual case of pseudo-Meigs syndrome with a uterine leiomyoma and an elevated CA125 level. CTA was used for the detection and ligation of the parasitic blood supply (in this case, mainly the blood vessels of the greater omentum). Surgeons should be aware of the malignant potential of this tumor and plan for the optimal surgical procedure, which would lead to a more appropriate treatment strategy.

\section{Declarations}

\section{Acknowledgments}

Patient consent was obtained for publication of his case record and specimen photograph.

\section{Grant support}

This study was supported by the Shanghai Municipal Key Clinical Specialty (No. Shslczdzk06302)

\section{Conflict of interest statement}

The authors declare that there are no conflicts of interest.

\section{Authors' contributions}

YW Wang-conceived the study, did literature search and coordinated preparation of the manuscript for submission.

Q Fan, Z Qian, J Wang-helped in literature search and in preparation of the manuscript.

Y Li, YD Wang,-reviewed and edited the manuscript for final submission.

All authors read and approved the manuscript.

\section{Availability of data and materials}

All data generated or analysed during this study are included in this published article.

\section{Ethics approval and consent to participate}

The ethics committee of International Peace Maternity \& Child Health Hospital approved this study. Study participant provided written informed consent prior to study enrollment. 


\section{Consent for publication}

consent to publication have be obtained from study participant.

\section{Competing interests}

None declared.

\section{References}

1. Marsh EE, Ekpo GE, Cardozo ER, et al. Racial differences in fibroid prevalence and ultrasound findings in asymptomaticyoung women (18-30 years old): a pilot study[J]. Fertil Steril, 2013,1951e7.

2. Babacan A, Kizilaslan C, Gun I, et al. Discussion-CA 125 and other tumor markers in uterine leiomyomas and their association with lesion characteristics[J]. International Journal of Clinical Experimental Medicine. 2014;7:1078-83.

3. Commandeur AE, Styer AK, Teixeira JM. Epidemiological and genetic clues for molecular mechanisms involved in uterineleiomyoma development and growth[J]. Hum Reprod Update, 2015.

4. Quade BJ, Wang TY, Sornberger K, et al. Molecular pathogenesis of uterine smooth muscle tumors from transcriptional profiling[J]. Genes Chromosomes Cancer. 2004;40:97-108.

5. Skubitz KM, Skubitz AP. Differential gene expression in leiomyosarcoma[J]. Cancer. 2003;98:102938.

6. Kawakubo N, Okido M, Tanaka R, et al. Pseudo-Meigs' syndrome associated with breast cancer metastasis to both ovaries: report ofa case[J]. Surg Today. 2010;40:1148-51.

7. Meigs JV, Armstrong SH, Hamilton $\mathrm{HH}$. A further contribution to the syndrome offi broma of the ovary with fluid in the abdomen and chest, Meigs' syndrome[J]. Am JObstet Gynecol. 1943;46:19-37.

8. Schmitt R, Weichert W, Schneider W, et al. Pseudo-pseudo Meigs' syndrome[J]. Lancet. 2005;366:1672.

9. Meigs JV. Pelvic tumors other than fibromas of the ovary with ascites and hydrothorax[J]. Obstet Gynecol. 1954;3:471-86.

10. Kyo K, Maema A, ShirakawaM, et al. Pseudo-Meigs' syndrome secondary to metachronous ovarian metastases from transverse colon cancer[J]. World J Gastroenterol. 2016;22:4604-9.

11. Kuo-Chien T, Ji-Hong H, Tsu-Lan W, et al. Elevation of CA 19 - 9 and Chromogranin A, in Addition to CA 125, Are Detectable in Benign Tumors in Leiomyomas and Endometriosis[J]. J Clin Lab Anal. 2017;21:71-6.

12. Yaguchi A, Ban K, Koshida Y, et al. Pseudo-Meigs Syndrome Caused by a Giant Uterine Leiomyoma with Cystic Degeneration: A Case Report[J]. J Nippon Med Sch. 2020;87:80-6.

13. Dong R, Jin C, Zhang Q. el al. Cellular leiomyoma with necrosis and mucinous degeneration presenting as pseudo-Meigs' syndrome with elevated CA125[J]. Oncol Rep. 2015;33:3033-7. 
14. Buckshee K, Dhond AJ, Mittal S, et al. Pseudo-Meigs' Syndrome Secondary to Broad Ligament Leiomyoma: A Case Report[J]. Asia-Oceania Journal of Obstetrics Gynaecology. 1990;16:201-5.

15. Oguma T, Yamasaki N, Nakanishi K, et al. Pseudo-Meigs' syndrome associated with hydropic degenerating uterine leiomyoma: A case report[J]. Journal of Obstetrics Gynaecology Research. 2014;40:1137-40.

16. Styer AK, Rueda BR. The Epidemiology and Genetics of Uterine Leiomyoma[J]. Best Practice and Research: Clinical Obstetrics and Gynaecology. 2016, 34: 3-12.

17. Kebapci M, Aslan O, Kaya T, et al. Pedunculated uterine leiomyoma associated with pseudo-Meigs' syndrome and elevated Ca-125 level: Ct features[J]. Eur Radiol. 2002;12:127-9.

18. Weinrach DM, Wang KL, Keh P, et al. Pathologic quiz case: a 40-year-old woman with a large pelvic mass, ascites, massive right hydrothorax, and elevated Ca125[J]. Arch Pathol Lab Med. 2004;128:933-4.

19. Ricci G, Inglese S, Candiotto A, et al. Ascites in puerperium: a rare case of atypical pseudo-Meigs' syndrome complicating the puerperium[J]. Arch Gynecol Obstet. 2009;280:1033-7.

20. Yip HK, Huang LW, Lin YH, et al. Massive ascites caused by a large pedunculated subserosal uterine leiomyoma that has feeding arteries from peripheral tissues and exhibits elevated Ca125: a case report[J]. J Obstet Gynaecol. 2014;34:107.

21. Niloff JM, Knapp RC, Schaetzl E, et al. CA-125 antigen levels in obstetric and gynecologic patients. Obstet Gynecol. 1984;64:703-7.

22. Yin $\mathrm{H}$, Li XH, Xu HM, et al. Pseudo-Meigs' syndrome secondary to bilateral ovarian endometrioid carcinomas[J]. Int JGynaecol Obstet. 1999;66:293-5.

23. Abramov Y, Anteby SO, Fasouliotis SJ, et al. Markedly elevated levels of vascular endothelial growth factor, fibroblast growth factor, and interleukin 6 in Meigs syndrome[J]. Am JObstet Gynecol. 2001;184:354-5.

24. IshikoO YH, SumiT, et al. Vascular endothelial growth factor levels in pleural and peritoneal fluid in Meigs' syndrome[J]. Eur JObstet Gynecol Reprod Biol. 2001;98:129-30.

25. Migishima $F$, Jobo $T$, Hata $H$, et al. Uterine leiomyoma causing massive ascites and left pleural effusion with elevated Ca 125: a case report[J]. J Obstet Gynaecol Res. 2000;26:283-7.

26. Roques F, Sanchez B, Bucher B, et al. Role of pre-operative assessment in the surgical management of leiomyoma extended to the right heart chambers: a compendium of information from isolated reports[J]. Eur J Cardiothorac Surg. 2001;19:522-4.

27. Kingdom JC, Hobson SR, Murji A, et al. Minimizing surgical blood loss at cesarean hysterectomy for placenta previa with evidence of placenta increta or placenta percreta: the state of play in 2020. Am J Obstet Gynecol. 2020;223:322-9.

28. He H, Yao M, Wu Y, et al. Difination-Highly elevated serum CA-125 levels in patients with nonmalignant gynecological diseases[J]. Arch Gynecol Obstet. 2011;283:123-6. 
29. Kil K, Chung E, Pak J, et al. Usefulness of CA125 in the differential diagnosis of uterine adenomyosis and myoma[J]. European Journal of Obstetrics Gynecology Reproductive Biology. 2015;185:131-5.

30. Kizilaslan C, Gun I, Muhcu M, et al. Discussion-CA 125 and other tumor markers in uterine leiomyomas and their association with lesion characteristics[J]. International Journal of Clinical Experimental Medicine. 2014;7:1078-83.

31. Bast RC Jr, Badgwell D, Lu Z, et al. New tumor markers: CA 125 and beyond[J]. Int J Gynecol Cancer. 2005;15:274-81.

32. Yilmaz N, Sahin I, Kilic S, et al. Assessment of the predictivity of preoperative serum CA 125 in the differential diagnosis of uterine leiomyoma and uterine sarcoma in the Turkish female population[J]. Eur J Gynaecol Oncol. 2009;30:412-4.

33. Tolman CJ, Vaid T, Schreuder HW.Extremely elevated CA-125 in benign ovarian disease due to stretch of the peritoneum[J].BMJ Case Rep. 2012, 27:bcr2012006664.

34. Bottoni P, Scatena R. The role of Ca 125 as tumor Marker: Biochemical and Clinical aspects[J]. Adv Exp Med Biol. 2015;867:229-44.

35. Zhang P, Zhang C, Hao J, et al. Use of X-chromosome inactivation pattern to determine the clonal origins of uterine leiomyoma and leiomyosarcoma[J]. Hum Pathol. 2006;37:1350-6.

36. Glorie N, Baert T, Van Den Bosch T, et al. Circulating protein biomarkers to differentiate uterine sarcomas from leiomyomas[J]. Anticancer Res. 2019;39:3981-9.

37. Kido A, Togashi K, Koyama T, et al. Diffusely enlarged uterus: evaluation with MR imaging. Radiographics[J]. 2003;23:1423-39.

\section{Figures}




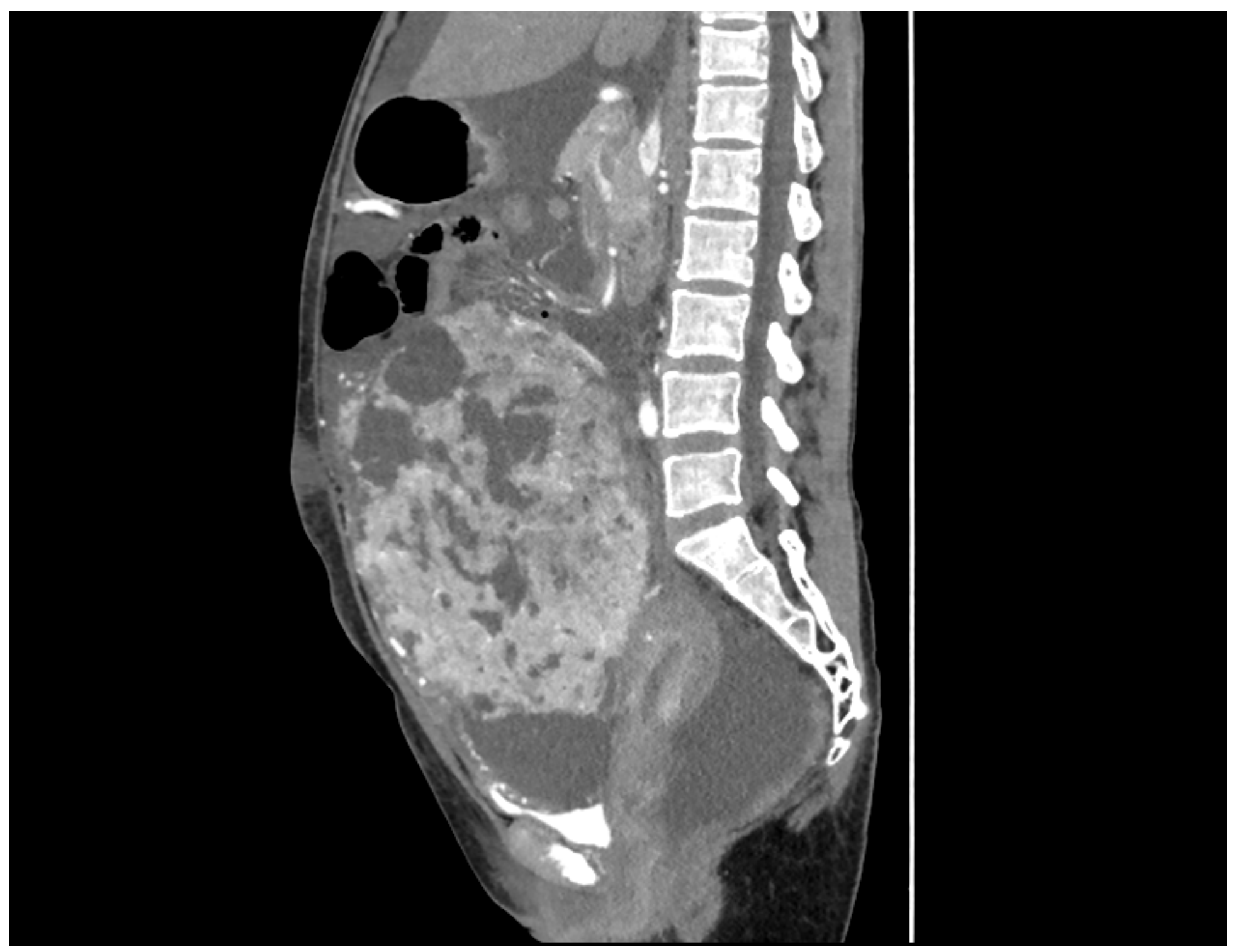

Figure 1

Enhanced CT of the abdomen/pelvis showing a large preperitoneal mass joined to the uterus. 


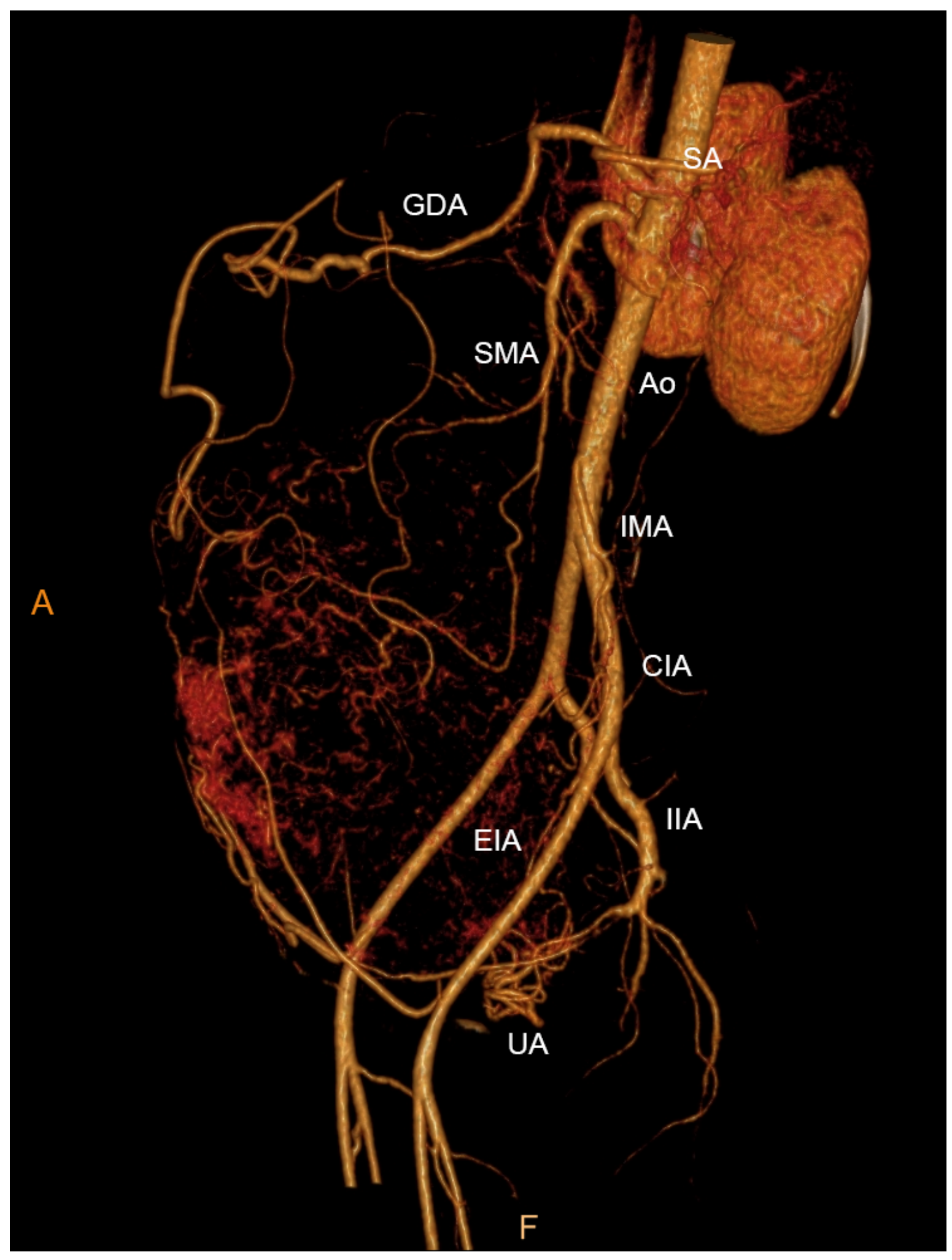

\section{Figure 2}

CTA showing an abundant blood supply surrounding the mass. AA, abdominal artery; CIA, common iliac artery; EIA, external iliac artery; GDA, gastroduodenal artery; IIA, internal iliac artery; IMA, inferior mesenteric artery; SA, splenic artery; SMA, superior mesenteric artery; UA, uterine artery. 

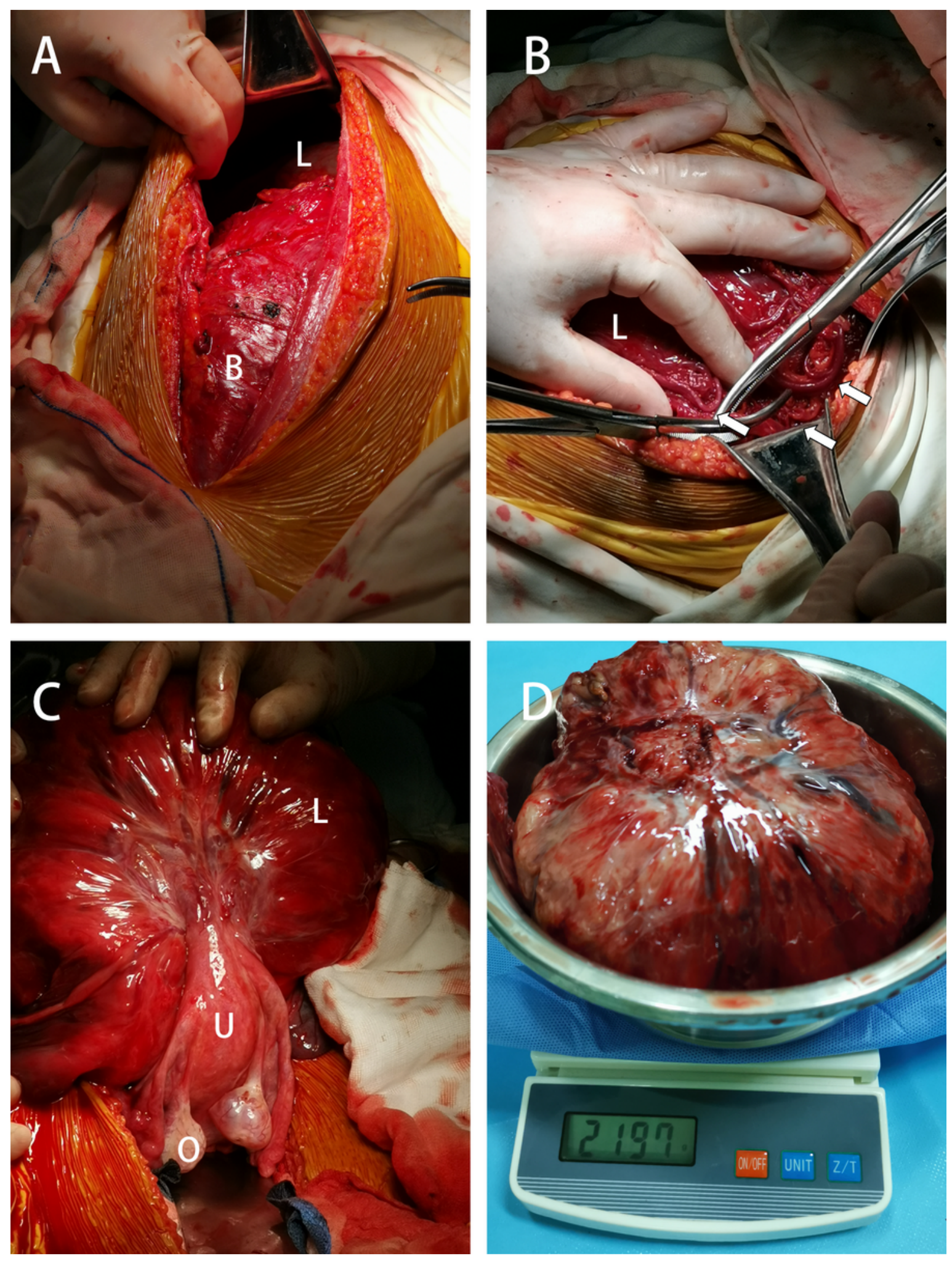

\section{Figure 3}

Intraoperative findings. (A) bladder with a dense adhesion and mass, which extended $3 \mathrm{~cm}$ below the umbilicus. (B) engorged vessels of the omentum majus that appeared along the surface of the mass. (C) lifted and turned mass showing a pedicle attached to the uterine fundus. (D) resected mass along with its pedicle stump. 

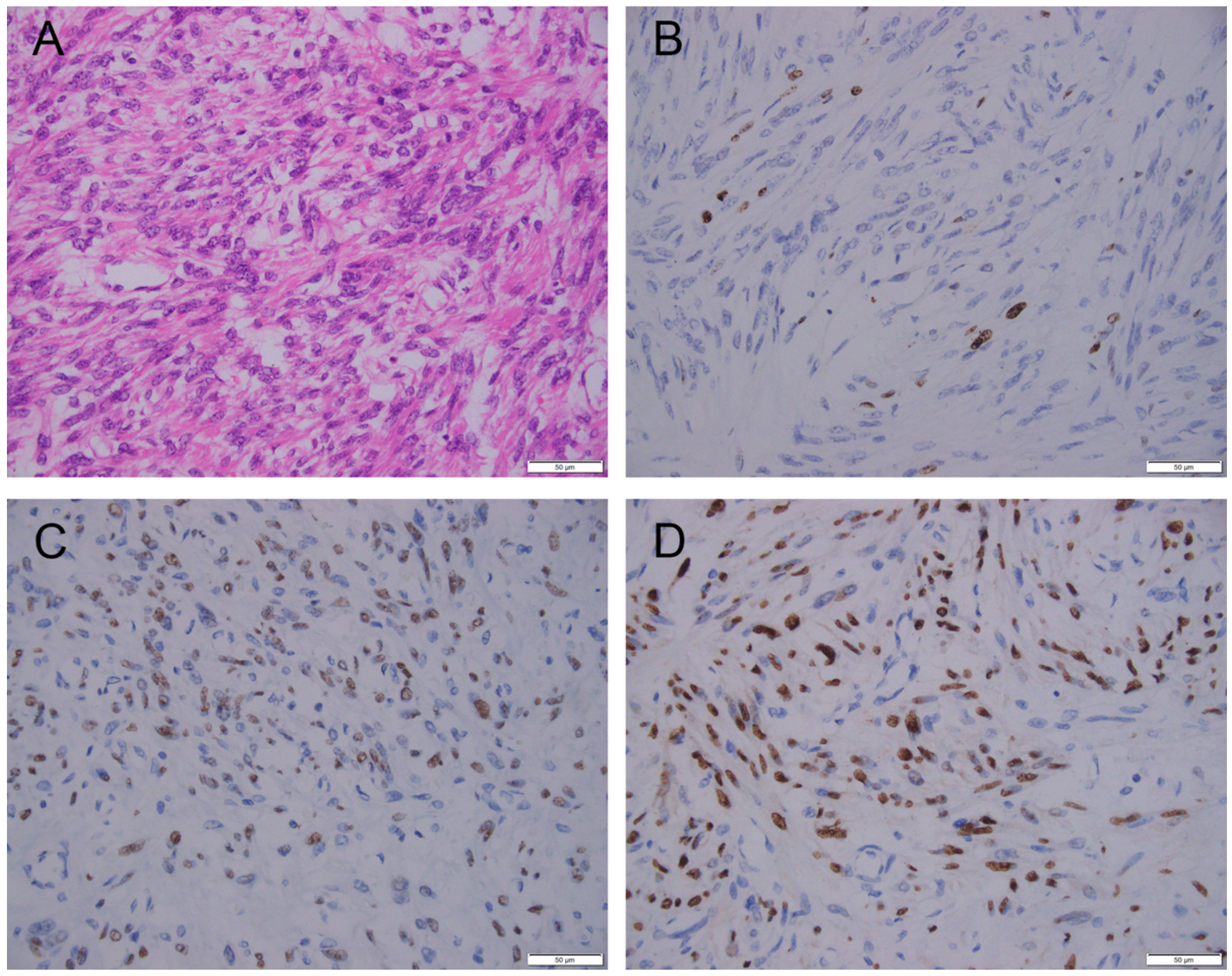

\section{Figure 4}

Representative immunohistochemical images of the leiomyoma. (A) H\&E staining. (B) Ki67 staining. (C) estrogen receptor staining. (D) progesterone receptor staining. Scale bar: $50 \mu \mathrm{m}$. 